\title{
ANGIOTOMOGRAFIA COMPUTADA EN PEDIATRIA: EXPERIENCIA EN UN HOSPITAL PEDIATRICO
}

\author{
Dra. Adriana Merchak A.
}

Servicio de Radiología Hospital Luis Calvo Mackenna. Santiago, Chile.

\section{COMPUTED ANGIOTOMOGRAPHY IN PEDIATRICS: EXPE- RIENCE IN A CHILDRE'S HOSPITAL}

\begin{abstract}
Assessment of cardiovascular diseases has proved to have a growing relevance in radiologic diagnosis, mainly due to the advancements in helicoidal computed tomography and magnetic resonance imaging.

Computed angiotomography is a highly accurate and useful diagnostic tool in pediatrics, basically for its short patient-study time and its high spatial resolution. Thus, conventional diagnostic angiography seems to have been ranked second.

This study presents the author's experience in the diagnosis of cardiovascular diseases using plain helicoidal computed tomography in a children's hospital belonging to the Public Health Care System.
\end{abstract}

Key words: Computed angiotomography, cardiovascular pathology, Pediatrics.

Resumen: La evaluación de la patología cardiovascular cobra cada vez mayor relevancia en el diagnóstico radiológico, fundamentalmente por los avances en las técnicas de tomografía computada helicoidal y resonancia magnética.

La angiotomografía computada es un método de gran rendimiento y utilidad diagnóstica en pediatría, principalmente por sus cortos tiempos de adquisición y su alta resolución espacial. Los grandes avances en esta técnica han desplazado a la angiografía convencional diagnóstica a un segundo lugar.

En este trabajo se presenta la experiencia del autor en el diagnóstico de las patologías cardiovasculares usando tomografía computada helicoidal simple, en un hospital pediátrico inserto en el Sistema Público de Salud.

Palabras clave: Angiotomografía computada, Patología cardiovascular, Pediatría.

Merchak A, y cols. Angiotomografía computada en pediatría: Experiencia en un hospital pediátrico. Rev Chil Radiol 2008; 14: 73-79.

Correspondencia: Dra. Adriana Merchak A.

adrianamerab@yahoo.com

\section{Introducción}

El estudio de la patología cardiovascular y su seguimiento funcional tradicionalmente han sido realizados con angiografía convencional, lo que implica intervención y un alto costo de radiación para el niño.

El avance y desarrollo de técnicas de angioresonancia (AngioRM) y angiotomografía computada (AngioTC) ha permitido su utilización en el diagnóstico de variadas patologías cardiovasculares de la infancia, tradicionalmente en manos de médicos no radiólogos, lo que constituye un desafío para el radiólogo, como lo avala la literatura ${ }^{(1)}$.

El gold standard en el estudio de estas patologías ha sido la angiografía convencional, con una dosis de radiación mucho mayor que la AngioTC. La AngioRM, igualmente útil, presenta limitaciones de disponibilidad y costo en nuestro medio y además habitualmente requiere de anestesia general en el niño, debido a sus largos tiempos de adquisición. Es por ésto que la AngioTC aparece como un examen de primera línea para uso en niños, ya que es muy rápido y otorga imágenes de excelente calidad.

La AngioTC de tórax permite visualizar no sólo el corazón y los grandes vasos sino también el pulmón y la vía aérea, aportando información adicional a otros métodos de diagnóstico cardiovascular. En el abdomen permite demostrar la anatomía vascular y la relación de las lesiones vasculares con los órganos vecinos. A diferencia de la AngioRM, en escasos segundos este examen permite valorar la patología a estudiar, utilizando medio de contraste no iónico que en general produce escasas reacciones adversas en los niños; además permite realizar estudios en pacientes con marcapasos y cirugías previas, ya que los artefactos metálicos producidos por los clips son menos significativos que en RM.

La AngioTC está indicada para la evaluación de cardiopatías congénitas, shunts, estudio de anillos vasculares, coartación aórtica, trauma aórtico, vasculitis, drenajes venosos pulmonares anómalos, secuestro pulmonar, hipertensión portal y estudio vascular, tanto hepático como renal en trasplantes ${ }^{(2)}$.

Existen múltiples trabajos que intentan protocolizar los estudios vasculares en TC, básicamente en adultos, que no necesariamente son aplicables en 
niños ya que existen muchos factores propios de la edad pediátrica que participan en la obtención de una buena imagen, entre los que destacan el volumen de contraste a utilizar que varía en forma importante en la literatura y la dificultad para lograr apnea ${ }^{(2-4,8)}$.

El objetivo de este trabajo es evaluar y mostrar la experiencia en el diagnóstico de la patología cardiovascular pediátrica mediante AngioTC en nuestro hospital, con una protocolización de los exámenes acorde a la tecnología disponible y comparar nuestra experiencia con los protocolos pediátricos extranjeros en relación a los parámetros utilizados, tanto en la imagen obtenida como en la calidad diagnóstica de los exámenes.

\section{Material y métodos}

El período de estudio incluyó desde enero a agosto del año 2005, considerando los pacientes derivados al Servicio de Rayos del Hospital Calvo Mackena para realizar una AngioTC.

Los exámenes se realizaron en un equipo de TC helicoidal GE de una hélice, con tiempo de giro de un segundo, modelo HiSpeed Xi, utilizando FOV de 18 a 20 para lactantes y de 22 a 24 en los niños más grandes. Se usó un grosor de corte lo más pequeño posible (1-5 mm) con intervalos de 1-5 mm, que permitieran disminuir la cantidad de radiación y el tiempo del examen; se realizó traslape de 50\% para reconstrucciones multiplanares.

Los estudios se planificaron con dosis de $\mathrm{kV}$ y $\mathrm{mA}$ semejantes a una TC de tórax y abdomen standard, variando durante el curso del estudio de acuerdo a resultados, con el objeto de disminuir dosis. Se utilizaron pitch de 1,5-1,8, dependiendo del tamaño del vaso a estudiar y de la contextura del niño. En todos los pacientes se midió la cantidad de radiación absorbida a través del dispositivo incorporado en el equipo, expresada en mGy.

Los exámenes se realizaron en apnea, con entrenamiento previo de los pacientes para conocer de antemano la capacidad máxima de apnea. Si el paciente no era capaz de retener el aire por el tiempo necesario, se protocolizaron dos fases de apnea con un breve intervalo inspiratorio de recuperación. En los lactantes, el examen se realizó con respiración tranquila.

Se utilizó contraste no iónico en dosis única de $1,5 \mathrm{cc} / \mathrm{kg}$, inyectando con bomba de infusión o técnica manual; los lactantes recibieron inyección manual debido al diámetro de sus vasos.

Se calculó el delay o tiempo de latencia necesario para el estudio del vaso en base a valores de estudios de adultos y se disminuyeron en forma empírica según la edad del paciente y el tipo de examen. No se dispuso de bolus tracking y no se realizó test bolus (inyección de prueba) con el objeto de no aumentar la dosis de radiación ni la dosis de contraste administrado. Posterior al examen, se valoró si el delay real fue adecuado para el estudio de la patología y se consignó el delay ideal para obtener óptimas imágenes en exámenes posteriores.

\section{Resultados}

Se realizaron estudios vasculares en 23 pacientes, 13 niñas y 10 niños, con diagnóstico clínico y exámenes complementarios de patología cardiovascular. Las edades de los pacientes estaban comprendidas entre los 3 meses y 18 años, con una mediana de 10 años.

Se realizaron 23 AngioTC: 11 de tórax, 10 de abdomen, 1 de cara y 1 de cerebro. Las patologías correspondieron a: 1 aneurisma de la arteria cerebral media, 1 malformación vascular cerebral, 1 tumor cerebral operado, 5 coartaciones aórticas (Figuras 1-3), 1 malformación aórtica, 1 tetralogía de Fallot, 1 secuestro pulmonar (Figura 4), 1 probable fístula arteriovenosa pulmonar, 1 síndrome del opérculo torácico, 5 cavernomatosis portales (Figura 5), 2 casos de hipertensión portal y 3 trasplantes hepáticos para valoración de estenosis de arteria hepática y vena porta (Figuras 6 y 7), como se resume en la Tabla I.

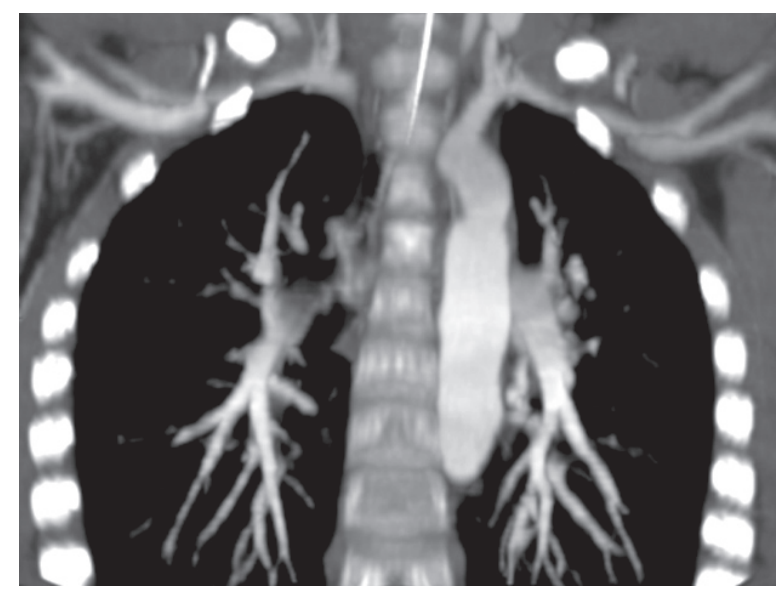

Figura 1. Reconstrucción coronal de coartación aórtica.

Figura 2 . Reconstrucción 3D de coartación aórtica.

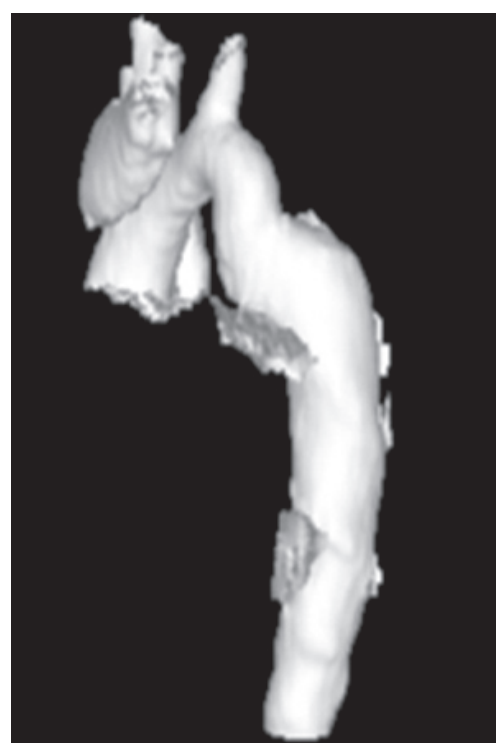




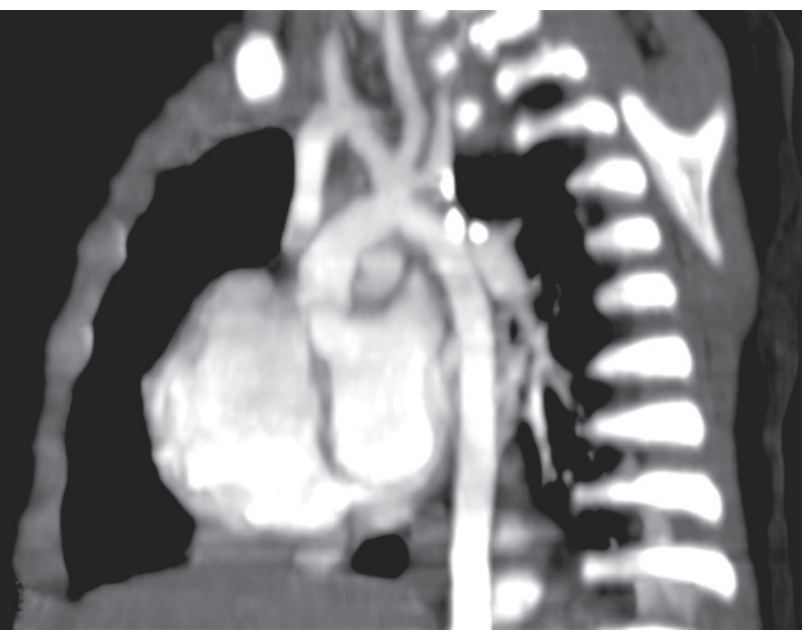

Figura 3. Reconstrucción coronal oblicua de coartación aórtica operada.

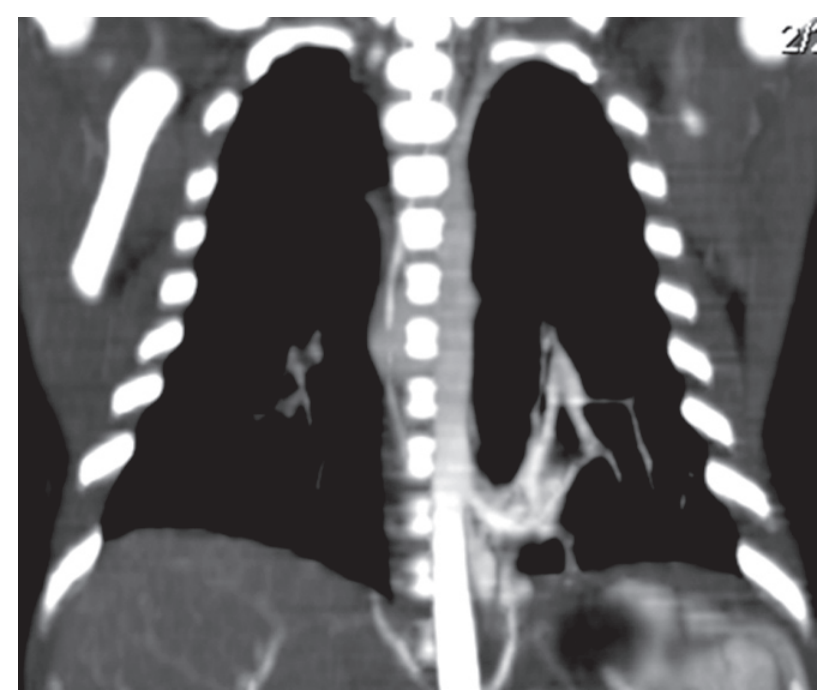

Figura 4. Reconstrucción coronal de secuestro pulmonar con 2 arterias nutricias emergiendo de aorta torácica y abdominal.

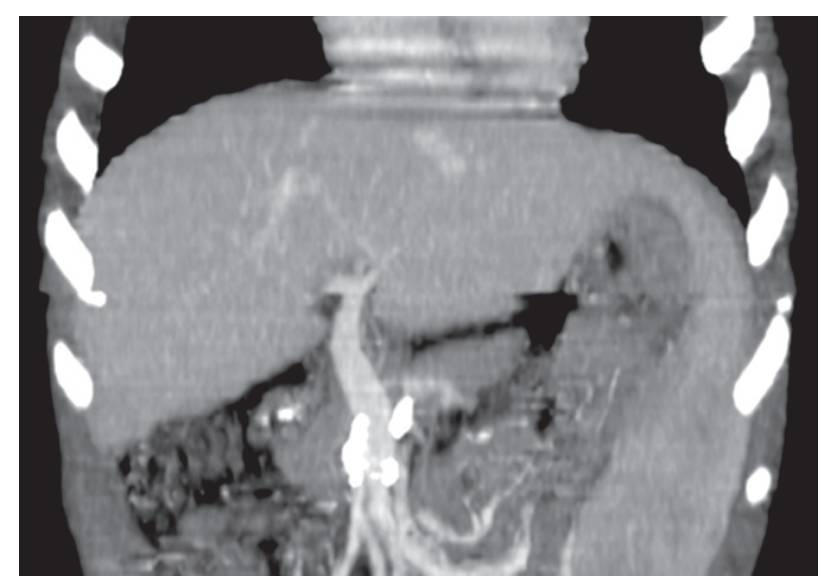

Figura 5. Reconstrucción coronal para visualización de shunt de Rex permeable.

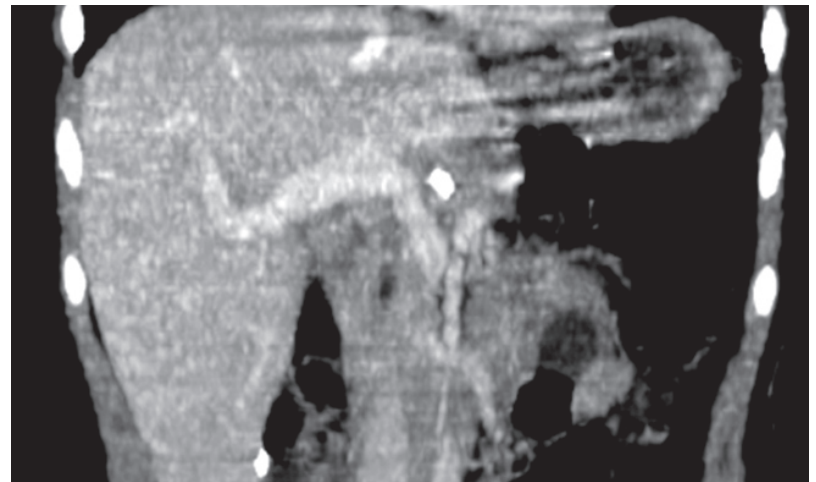

Figura 6. Reconstrucción coronal para demostrar trayecto de vena porta.

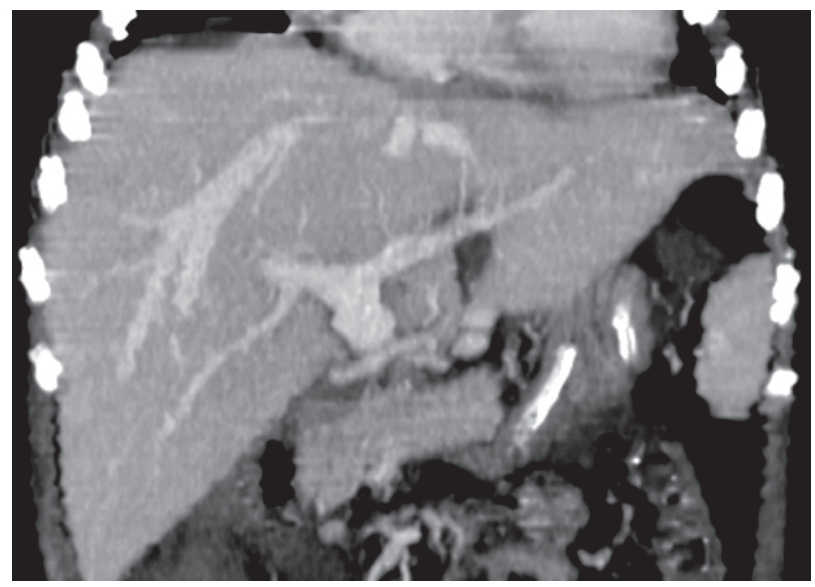

Figura 7. Reconstrucción coronal para demostrar trayecto de ambas ramas de vena porta.

En todos los pacientes se inyectó medio de contraste no iónico $(320 \mathrm{mg} / \mathrm{cc}$ ) en dosis de $1,5 \mathrm{cc} / \mathrm{kg}$ y se obtuvo buen contraste vascular. No hubo reacciones adversas en el grupo estudiado.

Para la administración del contraste, en el $97 \%$ de los niños se utilizó vía periférica con agujas 22G, inyectando con bomba de infusión en el $91,3 \%$ de los casos y en forma manual en el $8,7 \%$. En un paciente falló la inyección manual de contraste por ruptura de la vena, lográndose inyectar sólo parcialmente el contraste calculado. Se utilizó el antebrazo opuesto al vaso a estudiar en el caso de AngioTC de tórax, para así disminuir los artefactos por contraste: antebrazo derecho para estudiar la aorta y antebrazo izquierdo para la arteria pulmonar. La velocidad de infusión de contraste varió entre $1,5 \mathrm{cc} / \mathrm{seg}$ y $3 \mathrm{cc} / \mathrm{seg}$, siendo la menor de ellas utilizada en la infusión manual en lactantes (Tabla I).

Los primeros pacientes se realizaron con dosis de Kv y mA para TC de tórax y abdomen standard (120 Kv y 100-150 mA) y posteriormente se fueron variando los valores de $\mathrm{Kv}$ y $\mathrm{mA}$, dentro de los rangos que la máquina permitió, logrando disminuir la dosis total de radiación absorbida sin perder información diagnóstica. El mA se pudo bajar entre 70 y 130 , 
alcanzando en la mayoría de los pacientes valores menores o iguales a $96 \mathrm{~mA}$, como se observa en la Tabla II. El rango de variación del $\mathrm{mA}$ utilizado fue de 70 a 200. La disminución de Kv se pudo realizar sólo en un paciente, logrando buen contraste de las estructuras vasculares a estudiar, sin que el aumento del ruido fuera significativo en la imagen obtenida.

El grosor de corte utilizado varió de 1 a $5 \mathrm{~mm}$, dependiendo del tamaño del niño y del vaso a estudiar. En el $87 \%$ fue de $3 \mathrm{~mm}$, ya que el disminuir más el grosor aumentaba el tiempo de examen y la radiación absorbida, sin diferencias significativas en la calidad de la imagen diagnóstica.

El intervalo de corte varió de 1 a $5 \mathrm{~mm}$, utilizando $1,5 \mathrm{~mm}$ en el $74 \%$ de los casos. Las reconstrucciones multiplanares se realizaron con un traslape de $50 \%$, obteniendo imágenes de calidad diagnóstica.

El pitch utilizado varió de 1,5 a 1,8, intentando adecuar la velocidad de la mesa al tiempo de apnea del niño para así evitar al máximo la necesidad de utilizar más de una apnea. Cuando a pesar de aumentar el pitch no se logró ajustar al tiempo requerido, se decidió realizar el examen con 2 apneas y tiempos muy cortos de recuperación, entrenando previamente a cada paciente en la respiración, midiendo cuántos segundos duraba su apnea, para la planificación posterior del examen. En el $48 \%$ de los exámenes se utilizó pitch de 1,8 y en el 39\% pitch de 1,5.

El delay real utilizado varió en las AngioTC de tórax entre 0 y 10 segundos desde el inicio de la inyección del contraste y en las AngioTC de abdomen entre 2 y 12 segundos, dependiendo del vaso a estudiar (arterial, portal o venoso) y del lugar de infusión (periférico o central). El delay ideal registrado posterior a la evaluación del examen realizado se definió valorando la calidad de la imagen obtenida y fue semejante al real en el $78 \%$ de los casos, menor al utilizado en 4 pacientes y mayor al real en 2 .

La valoración de la calidad de la imagen obtenida se realizó considerando el grado de opacificación alcanzado, la visualización del vaso a estudiar y el ruido de la imagen obtenida.

\section{Discusión}

El estudio de la patología vascular en los niños ha sido realizado tradicionalmente con angiografía convencional. Este examen, considerado por muchos años como el gold standard para este estudio, es un examen invasivo y con un alto costo de radiación en los niños ${ }^{(1)}$.

En los últimos años ha aparecido la AngioTC como un examen atractivo para el estudio vascular en niños, ya que es muy rápido y obtiene imágenes de excelente calidad. Permite visualizar no sólo los

\begin{tabular}{|c|c|c|c|c|c|c|c|c|c|c|}
\hline Paciente & $\begin{array}{l}\text { Edad } \\
\text { (años) }\end{array}$ & Sexo & $\begin{array}{l}\text { Peso } \\
(\mathrm{kg})\end{array}$ & Contraste & $\begin{array}{l}\text { Dosis } \\
\text { (cc) }\end{array}$ & Aguja & Vía & $\begin{array}{l}\text { Tipo de } \\
\text { infusión }\end{array}$ & $\begin{array}{l}\text { Velocidad } \\
\text { infusión }\end{array}$ & Diagnóstico \\
\hline 1 & 12 & $M$ & 32 & $\mathrm{NI}$ & 50 & 22 & $P$ & $\mathrm{~B}$ & 3 & Coartación aórtica \\
\hline 2 & 8 & $\mathrm{~F}$ & 33 & $\mathrm{NI}$ & 50 & 22 & $\mathrm{P}$ & M & 1,5 & Secuestro pulmonar \\
\hline 3 & 10 & $\mathrm{~F}$ & 34 & $\mathrm{NI}$ & 53 & 22 & $P$ & $\mathrm{~B}$ & 3 & Aneurisma cerebral \\
\hline 4 & 9 & $M$ & 32 & $\mathrm{NI}$ & 74 & 22 & $\mathrm{P}$ & $\mathrm{B}$ & 3 & Coartación aórtica \\
\hline 5 & 0,25 & M & 4 & $\mathrm{NI}$ & 8 & 24 & $\mathrm{P}$ & M & 1,5 & Coartación aórtica \\
\hline 6 & 13 & $M$ & 48 & $\mathrm{NI}$ & 80 & 22 & $P$ & $\mathrm{~B}$ & 3 & Coartación aórtica \\
\hline 7 & 12 & $M$ & 45 & $\mathrm{NI}$ & 80 & 22 & $P$ & $\mathrm{~B}$ & 3 & Tetralogía de Fallot \\
\hline 8 & 13 & M & 55 & $\mathrm{NI}$ & 100 & 22 & $\mathrm{P}$ & $\mathrm{B}$ & 3 & Síndrome Marfan \\
\hline 9 & 11 & $\mathrm{~F}$ & 31 & $\mathrm{NI}$ & 50 & 22 & $P$ & $\mathrm{~B}$ & 3 & Fístula AV pulmonar \\
\hline 10 & 13 & $M$ & 34 & $\mathrm{NI}$ & 50 & 22 & $\mathrm{P}$ & $\mathrm{B}$ & 3 & Trasplante hepático \\
\hline 11 & 10 & $\mathrm{~F}$ & 29 & $\mathrm{NI}$ & 50 & 22 & $P$ & $\mathrm{~B}$ & 3 & Hipertensión portal \\
\hline 12 & 11 & $\mathrm{~F}$ & 36 & $\mathrm{NI}$ & 50 & 22 & $\mathrm{P}$ & $\mathrm{B}$ & 3 & Trasplante hepático \\
\hline 13 & 9 & $M$ & 27 & $\mathrm{NI}$ & 42 & 22 & $P$ & $\mathrm{~B}$ & 3 & Hipertensión portal \\
\hline 14 & 10 & $M$ & 27 & $\mathrm{NI}$ & 42 & 22 & $P$ & $\mathrm{~B}$ & 3 & Cavernomatosis porta \\
\hline 15 & 2 & $\mathrm{~F}$ & 8 & $\mathrm{NI}$ & 12 & 22 & $P$ & $\mathrm{~B}$ & 2 & Cavernomatosis porta \\
\hline 16 & 14 & $M$ & 30 & $\mathrm{NI}$ & 45 & 22 & $P$ & $\mathrm{~B}$ & 3 & Trasplante hepático \\
\hline 17 & 9 & $M$ & 30 & $\mathrm{NI}$ & 45 & 22 & C & $\mathrm{B}$ & 3 & Estallido aórtico \\
\hline 18 & 12 & M & 70 & $\mathrm{NI}$ & 100 & 22 & $\mathrm{C}$ & B & 3 & $\begin{array}{l}\text { Tu cerebral op Ensan } \\
\text { chamiento mediastínico }\end{array}$ \\
\hline 19 & 7 & $M$ & 21 & $\mathrm{NI}$ & 32 & 22 & $P$ & $\mathrm{~B}$ & 3 & Coartación aórtica \\
\hline 20 & 18 & $\mathrm{~F}$ & 53 & $\mathrm{NI}$ & 80 & 22 & $\mathrm{P}$ & $\mathrm{B}$ & 3 & $\begin{array}{l}\text { Síndrome opérculo } \\
\text { torácico }\end{array}$ \\
\hline 21 & 10 & $M$ & 28 & $\mathrm{NI}$ & 42 & 22 & $\mathrm{P}$ & B & 3 & Cavernomatosis porta \\
\hline 22 & 14 & M & 45 & $\mathrm{NI}$ & 70 & 22 & $\mathrm{P}$ & $\mathrm{B}$ & 3 & $\begin{array}{l}\text { Osteosarcoma man- } \\
\text { díbula }\end{array}$ \\
\hline 23 & 2 & $\mathrm{~F}$ & 8 & $\mathrm{NI}$ & 12 & 22 & $P$ & $\mathrm{~B}$ & 2 & Cavernomatosis porta \\
\hline
\end{tabular}


Tabla II. Parámetros utilizados en los exámenes.

\begin{tabular}{|c|c|c|c|c|c|c|c|c|c|c|}
\hline Paciente & kV & $\mathrm{mA}$ & $\begin{array}{l}\text { Dosis } \\
\text { (mGy) }\end{array}$ & $\begin{array}{l}\text { Grosor } \\
\text { corte }\end{array}$ & Intervalo & Pitch & $\begin{array}{l}\text { Delay } \\
\text { real }\end{array}$ & $\begin{array}{l}\text { Delay } \\
\text { ideal }\end{array}$ & Apnea & AngioTc \\
\hline 1 & 120 & 110 & 4,4 & 3 & 3 & 1,8 & 7 & 8 & 2 & Tórax \\
\hline 2 & 120 & 80 & 6,4 & 3 & 3 & 1,8 & 6 & 6 & 2 & Tórax \\
\hline 3 & 120 & 150 & 8,4 & 1 & 1 & 1,5 & 4 & 4 & - & Cerebro \\
\hline 4 & 120 & 150 & 6,3 & 3 & 1,5 & 1,7 & 3 & 3 & 2 & Tórax \\
\hline 5 & 120 & 100 & 4,8 & 3 & 1 & 1,5 & 0 & 0 & $\begin{array}{l}\text { respiración } \\
\text { suave }\end{array}$ & Tórax \\
\hline 6 & 120 & 200 & 22,8 & 3 & 1,5 & 1,8 & 6 & 6 & 1 & Tórax \\
\hline 7 & 120 & 150 & 9,5 & 3 & 1,5 & 1,5 & 7 & 7 & 1 & Tórax \\
\hline 8 & 120 & 150 & 5,9 & 5 & 2,5 & 1,8 & 13 & 13 & 1 & Tórax y abd \\
\hline 9 & 120 & 100 & 4,8 & 3 & 1,5 & 1,8 & 4 & 4 & 2 & Tórax \\
\hline 10 & 120 & 100 & $4,0 \times 2$ & 3 & 1,5 & 1,8 & 11 & 9 & 2 & Abdomen \\
\hline 11 & 120 & 130 & $6,2 \times 2$ & 3 & 1,5 & 1,5 & 6 & 20 & 1 & Abdomen \\
\hline 12 & 80 & 80 & 1,1 & 3 & 1,5 & 1,5 & 7 & 5 & 1 & Abdomen \\
\hline 13 & 120 & 100 & $4,5 \times 2$ & 3 & 1,5 & 1,6 & 6 & 6 & 2 & Abdomen \\
\hline 14 & 120 & 100 & $4,5 \times 2$ & 3 & 1,5 & 1,6 & 5 & 5 & 2 & Abdomen \\
\hline 15 & 120 & 70 & $7 \times 2$ & 3 & 1,5 & 1,5 & 2 & 4 & 1 & Abdomen \\
\hline 16 & 120 & 100 & $3,6 \times 2$ & 3 & 1,5 & 1,8 & 12 & 12 & 1 & Abdomen \\
\hline 17 & 120 & 80 & 3,2 & 3 & 1,5 & 1,8 & 5 & 5 & 2 & Tórax \\
\hline 18 & 120 & 130 & 5,1 & 5 & 5 & 1,8 & 7 & 7 & 1 & Tórax \\
\hline 19 & 120 & 80 & 3,8 & 3 & 1,5 & 1,5 & 10 & 10 & 1 & Tórax \\
\hline 20 & 120 & 80 & 3,2 & 3 & 1,5 & 1,8 & 10 & 10 & 1 & Tórax \\
\hline 21 & 120 & 90 & $3,6 \times 2$ & 3 & 1,5 & 1,8 & 15 & 15 & 2 & Abdomen \\
\hline 22 & 120 & 100 & $10 \times 2$ & 3 & 1,5 & 1,5 & 20 & 20 & $\begin{array}{l}\text { respiración } \\
\text { suave }\end{array}$ & Cara \\
\hline 23 & 120 & 85 & $8,5 \times 2$ & 3 & 1,5 & 1,5 & 4 & 4 & 1 & Abdomen \\
\hline
\end{tabular}

vasos a estudiar sino también los órganos vecinos, aportando información adicional a la angiografía convencional. Utiliza dosis de contraste muy inferiores a la angiografía convencional: $1,5 \mathrm{cc} / \mathrm{kg}$ en este estudio y dosis habituales de $5-6 \mathrm{cc} / \mathrm{kg}$ en angiografía convencional, lo que posibilita repetir la inyección de contraste en caso de necesidad, sin alcanzar las altas dosis utilizadas convencionalmente ${ }^{(2)}$.

Al revisar la literatura nos encontramos con múltiples trabajos que han protocolizado la AngioTC en adultos pero que no son claros en relación con los niños ${ }^{(3-6)}$, ya que existen múltiples factores que intervienen en una adecuada opacificación de los vasos. Algunos han intentado protocolizar la AngioTC en niños ${ }^{(1,2)}$, evaluando los distintos factores involucrados en la opacificación de los vasos, entre los que destacan:

1. Localización del sitio de inyección: que puede ser periférico o central-lo que intervendrá directamente en la elección del delay- mientras más cercano al corazón menor será el delay a usar. En los pacientes con vía central se disminuye al menos en 3 segundos el delay elegido, obteniendo un buen contraste de los vasos. Es importante considerar el lado de la inyección del contraste, ya que si se va a estudiar la aorta debe ingresar por el antebrazo derecho y por el izquierdo si se estudia la arteria pulmonar, para disminuir artefactos producidos por el contraste en la zona.
2. Volumen de contraste: mientras más pequeño es el paciente, menor será la cantidad de contraste a inyectar. En la literatura se plantea el uso de 1,5 a $4 \mathrm{cc} / \mathrm{kg}$ de contraste yodado ${ }^{(1,2)}$; este estudio se realizó con dosis de $1,5 \mathrm{cc} / \mathrm{kg}$, con excelentes resultados, utilizando $100 \mathrm{cc}$ como dosis máxima.

3. Velocidad de inyección: debe ser la más uniforme posible, lo que se logra con el uso de bomba de infusión a velocidades de 2-3 cc/seg y en menor medida con la inyección manual, que logra velocidades máximas de 1,6 cc/seg utilizando presión constante.

4. Frecuencia cardíaca: se relaciona inversamente con el delay. La frecuencia varía con la edad (a menor edad mayor frecuencia cardíaca) y el grado de stress del niño previo al examen, lo que debe ser siempre evaluado antes de decidir el delay a utilizar. Niños muy ansiosos, en nuestra serie obligaron a disminuir el delay en al menos 2 segundos, debido a la taquicardia.

5. Anatomía de la patología a estudiar: es necesario conocer adecuadamente las alteraciones anatómicas y funcionales que pueden producirse en cardiopatías complejas, trasplantes, malformaciones pulmonares, conexiones quirúrgicas, shunts, etc.

6. Tipo de vaso a estudiar: el delay elegido variará según se trate de un vaso torácico o abdominal y arterial, portal o venoso. El delay para estudiar 
la arteria pulmonar es 1-2 segundos menor que para la aorta.

7. Respiración: la mejor calidad radiológica de una AngioTC se obtiene en apnea, lo que no siempre es posible en niños, planteando la necesidad de sedación en pacientes pequeños o la realización del examen con respiración tranquila, que si bien afecta directamente la calidad del examen no añade riesgos de anestesia o sedación. En este estudio no fue necesario utilizar anestesia en niños pequeños, realizando el examen con respiración tranquila que permitió obtener una calidad de imagen suficiente para el diagnóstico de la patología, aún cuando de inferior calidad al compararla con imágenes obtenidas con TC helicoidal multicorte. Realizamos AngioTC en apnea en todos aquellos niños capaces de obedecer órdenes simples (20/23 pacientes) y en los lactantes usamos respiración tranquila. En las AngioTC de tórax, la apnea se realizó en inspiración máxima, lo que permitió valorar adecuadamente el pulmón, situación que no se especifica en algunas publicaciones ${ }^{(2)}$.

8. Tipo de contraste: utilizamos contraste no iónico al igual que en otros estudios publicados ${ }^{(1,2,7,8)}$, ya que a pesar que las reacciones adversas en niños son menos frecuentes que en adultos, el uso de contraste no iónico ha demostrado disminución significativa de las reacciones adversas graves ${ }^{(9)}$.

En todos los niños se utilizó contraste isosmolar $320 \mathrm{mgl} / \mathrm{ml}$, sin necesidad de aumentar la dosis de yodo en los niños pequeños, ya que con éste se obtuvo buen contraste con mediciones de 150-200 UH en los vasos estudiados.

9. Velocidad de infusión: en la mayoría de los casos la velocidad fue de $3 \mathrm{cc} / \mathrm{seg}(83 \%)$, sin observar artefactos de movimiento ni molestias en realción con la inyección de contraste. Previo al examen se explicó a los pacientes los síntomas que podían presentar y se mantuvo una persona debidamente protegida al lado de cada niño, generalmente la madre, para darles confianza.

10. Delay: su adecuada elección se relaciona directamente con una buena opacificación de los vasos. En la literatura se analizan tanto el delay empírico como el test bolus y el bolus tracking, valorando las ventajas de éstos últimos ${ }^{(1,2)}$. Teniendo en cuenta los factores analizados previamente, en este estudio sólo pudimos utilizar delay empíricos que se acercaron bastante a los delay ideales, con buenos resultados. Mientras menor es el paciente, menor es el delay a usar y mientras más cercano al corazón sea el sitio de inyección, menor será el delay necesario, por lo que se debe elegir delay mayores cuando se trata de abdomen y menores cuando el estudio a realizar es de tórax. En esta serie, disminuimos en 3 segundos el delay elegido al usar vía venosa central. En general, para el estudio arterial se debe elegir un delay que permita la entrada del $40-50 \%$ de la dosis antes del inicio de la adquisición y para el estudio venoso, la entrada de al menos el $75 \%$ de ella.

Existen otros parámetros técnicos a considerar en estos estudios:

a. Tipo de TC helicoidal a utilizar: a mayor número de hélices mejor será la calidad de la imagen, menores los artefactos por movimiento y mínimo el tiempo de examen, con mayor dosis absorbida. Este estudio se realizó con un TC helicoidal de 1 hélice, que si bien no da la mejor calidad de imagen, permite imágenes de calidad suficiente para hacer el diagnóstico.

b. Tamaño del FOV: debe ser lo más pequeño posible en lactantes y algo mayor en niños escolares y adolescentes. No debemos olvidar que la disminución del FOV conlleva una mejor imagen pero produce una mayor radiación en los pacientes, por lo que sólo se debe usar FOV pequeños en lactantes.

c. Grosor de corte: depende del tamaño del niño y del vaso a estudiar. La literatura recomienda grosores entre 2,5 y $3,75 \mathrm{~mm}$, concordantes con los de nuestra serie.

d. Intervalos de reconstrucción: se usaron intervalos de $50 \%$ para reconstrucciones multiplanares y tridimensionales.

e. Kv y mA: se sabe que la disminución del Kv disminuye significativamente la dosis absorbida, ya que tiene una curva exponencial de disminución. Sólo en un paciente pudimos disminuir el Kv a 80, lo que disminuyó significativamente la dosis de radiación absorbida si se compara con el resto de los pacientes en que sólo pudimos modificar el mA. En la literatura se plantea el uso de 120 Kv para lactantes y $140 \mathrm{Kv}$ para niños mayores ${ }^{(1)}$, otros plantean $80 \mathrm{Kv}$ en menores de 1 año, 100 Kv entre 1 y 4 años y 100-120 Kv en mayores de 4 años ${ }^{(2)}$. Utilizamos dosis máximas de $200 \mathrm{~mA}$ y mínimas de $70 \mathrm{~mA}$, logrando disminuir la dosis absorbida en forma importante (Tabla II. Gráficos $1,2,3)$. El análisis de nuestro estudio arroja una curva de regresión lineal entre la disminución absoluta del mA y la disminución absoluta de la dosis de radiación en nuestros pacientes, lo que ilustra ampliamente la ventaja de reducir el $\mathrm{mA}$ para obtener una disminución importante de la dosis de radiación absorbida.

f. Pitch: la literatura recomienda realizar exámenes con pitch entre 1,5 y 2,0(1), para lo cual se debe conocer con exactitud la capacidad de apnea del niño, la longitud del segmento a estudiar y el grosor de corte mínimo necesario para no sobrepasar el valor de pitch a utilizar. Si se necesitan cortes más finos, una opción es realizar apneas repetidas, tal como se hizo con algunos de nuestros pacientes o utilizar sedación. Es claro que adquirir este tipo 
de exámenes sólo con respiración tranquila, en un TC helicoidal de una hélice da bastante artefacto de movimiento, lo que limita el rendimiento de los exámenes para valorar vasos muy finos, sobretodo en los niños más pequeños.

Gráficos 1, 2 y 3 . Visualización de valores de mA y dosis de radiación.

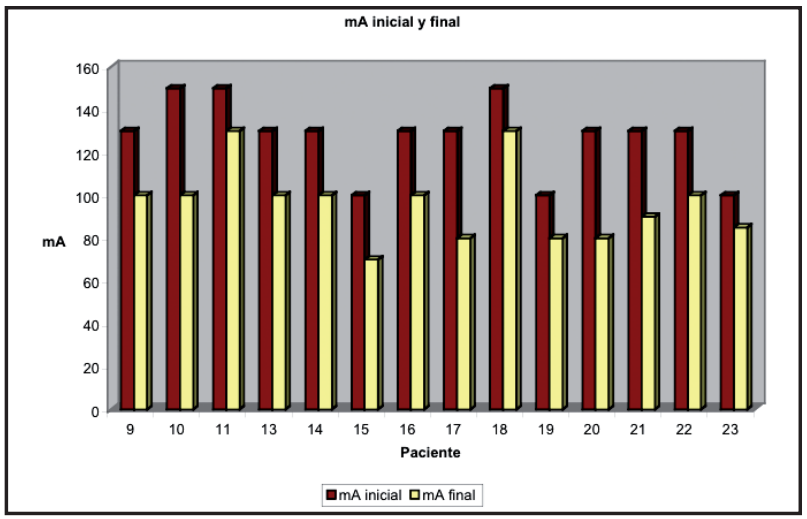

Gráfico 1. Variación entre $m A$ inicial y final.

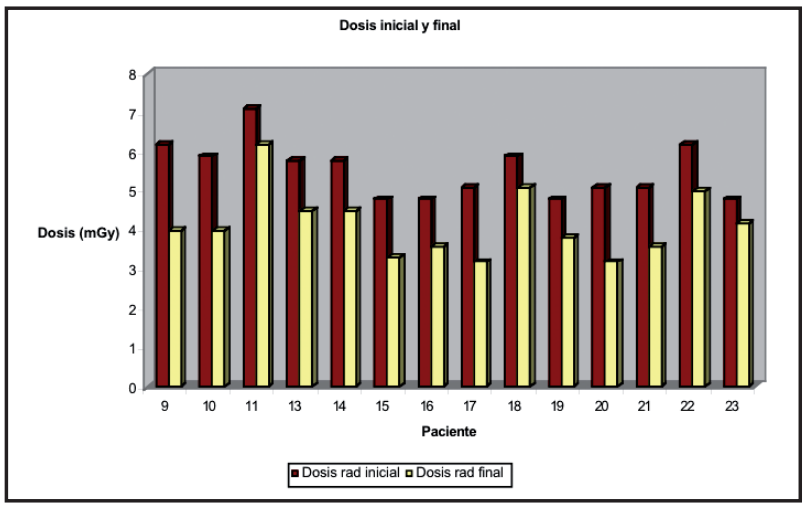

Gráfico 2. Variación obtenida entre dosis de radiación inicial y final, producida por la variación del $m A$.

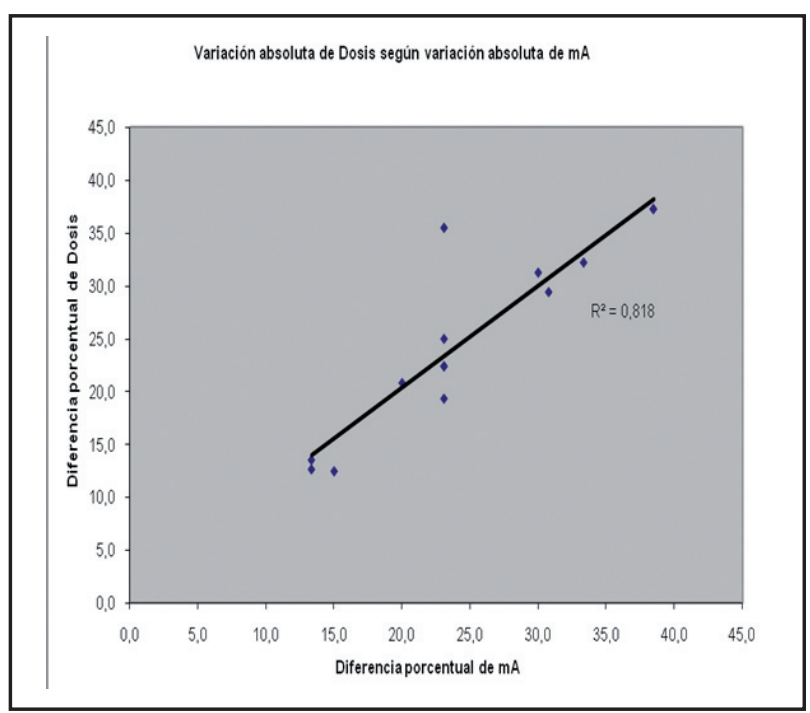

Gráfico 3. Correlación entre variación de $m A$ y variación de dosis.

\section{Conclusión}

Este estudio muestra la experiencia local en un hospital público, donde no se dispone de la tecnología óptima para realizar exámenes complejos. Sin embargo, controlando concienzudamente los parámetros descritos, con los recursos existentes se puede obtener estudios con imágenes de calidad suficiente que permitan llegar al diagnóstico planteado, evitando en la gran mayoría de los casos la necesidad de angiografía convencional y, decidir un tratamiento médico o quirúrgico.

La AngioTC es un estudio atractivo para ser utilizado en niños, que requiere conocer múltiples parámetros que afectarán la calidad de la imagen y por ende su interpretación. El familiarizarse con estos parámetros y ganar experiencia en la modificación de ellos logrando estudios satisfactorios, hará que la AngioTC sea utilizada por los radiólogos con más confianza en pacientes pediátricos.

La disponibilidad de TC helicoidal multicorte mejora sin duda las posibilidades de estudio, pero no debemos olvidar que aumenta la dosis absorbida.

\section{Bibliografía}

1. Cohen R, Frush D, Donnelly L. Data acquisition for pediatric CT angiography: problems and solutions. Pediatr Radiol 2000; 30: 813-822.

2. Frush D. Technique of Pediatric Thoracic CT Angiography. Radiol Clin N Am 2005; 43: 419-433.

3. Rubin GD. Three dimensional helical CT Angiography. Radiographics 1994; 14: 905-912.

4. Fishman EK. High resolution three dimensional imaging from sub-second helical CT data sets: applications in vascular imaging. AJR 1997; 169: 441-443.

5. Rubin GD, Dake MD, Semba CP. Current status of three dimensional spiral CT scanning for imaging the vasculature. Radiol Clin North Am 1995; 33: 51-70.

6. Zeman R, Silverman P, Vieco P. CT Angiography. AJR 1995; 165: 1.079-1.088.

7. Schaffler G, Sorantin E, Groell R et als. Helical CT Angiography with Maximum Intensity Projection in the Assessment of Aortic Coarctation After Surgery. AJR 2000; 175: 1.041-1.045.

8. Siegel M. Multiplanar and Three dimensional Multi Detector Row CT of Thoracic Vessels and Airways in the Pediatric Population. Radiology 2003; 229: 641650.

9. Cohan $\mathrm{R}$, Ellis $\mathrm{J}$. lodinated contrast material in uroradiology.Urol Clin North Am 1997; 24: 471-491. 